\title{
Estabilización de suelos con cenizas de carbón para uso como subrasante mejorada
}

\section{Stabilization of soils with coal ashes for use as an improved subgrade}

\author{
Olger Goñas Labajos ${ }^{1}$; Jhon Hilmer Saldaña Núñez ${ }^{2}$
}

\section{RESUMEN}

La baja capacidad de soporte que presentan los suelos de la ciudad de Chachapoyas, es desfavorable para ser usadas como subrasante por lo que este trabajo de investigación experimental, tuvo como objetivo evaluar la influencia que tiene un subproducto obtenido de la quema de carbón mineral y carbón vegetal (cenizas de carbón) proveniente de una industria ladrillera de la ciudad de Chachapoyas en el mejoramiento de las propiedades mecánicas de muestras de suelo. El estudio se inició tomando muestras de suelo de las cuadras ocho y nueve de calle Las Lomas, anexo 16 de Octubre a las que se les efectuó ensayos de: humedad natural, granulometría, límites de consistencia, compactación proctor estándar y capacidad de soporte (CBR) a cada muestra de suelo en estado natural. Las muestras adicionadas cenizas de carbón al 15\%,20\% y 25\% solo se le realizaron ensayos de límites de consistencia, compactación proctor estándar y capacidad de soporte (CBR) respectivamente; los resultados mostraron que el incremento de la capacidad de soporte de los suelos estudiados es directamente proporcional a los porcentajes de cenizas de carbón adicionados, llegando a la conclusión que las cenizas de carbón si mejoran la capacidad portante de los suelos de tipo $\mathrm{CH}$ y $\mathrm{OH}$ pero los porcentajes de cenizas adicionados no logran estabilizarlos según el manual de carreteras, suelos, geología, geotecnia y pavimentos, para ser usados como una subrasante mejorada.

Palabras clave: Estabilización de suelos, cenizas de carbón, subrasante.

\begin{abstract}
The low support capacity presented by the soils of the city of Chachapoyas, is unfavorable to be used as an subgrade so that this experimental research work, aims to assess the influence that a by-product obtained from the burning of coal and coal has Vegetable (coal ash) from a brick industry in the city of Chachapoyas in the improvement of the mechanical properties of soil samples. The study began by taking soil samples from blocks eight and nine of Las Lomas street, annex October 16, which were tested for: natural humidity, grain size, consistency limits, standard proctor compaction and support capacity (CBR ) to each soil sample in its natural state. The samples added 15\%, 20\% and 25\% coal ash were only tested for consistency limits, standard proctor compaction and support capacity (CBR) respectively; The results showed that the increase in the bearing capacity of the studied soils is directly proportional to the percentages of added carbon ashes, concluding that coal ashes do improve the bearing capacity of the soils of type $\mathrm{CH}$ and $\mathrm{OH}$ but the percentages of added ashes fail to stabilize them according to the manual of roads, soils, geology, geotechnics and pavements, to be used as an improved subgrade.
\end{abstract}

Keywords: Soil stabilization, coal ash, subgrade. 


\section{INTRODUCCIÓN}

El suelo que sostiene las cargas transmitidas por la superficie de rodadura de una vía debe encontrarse en condiciones tales, que sea competente para resistirlas, cuando este no es el caso las alternativas de solución son: cambiar el suelo por un material de préstamo (utilizar material de cantera cercanas al proyecto), aunque esto se considera una buena solución, por lo general tiene el inconveniente de alto costo debido al material de reemplazo; siendo la otra alternativa de realizar un método de estabilización para modificar sus propiedades mecánicas sin tener que eliminar el suelo existente(Peréz C. , 2014, pág. 16)

Muchos suelos debido a su baja capacidad de soporte y mala calidad no siempre cumplen con los requerimientos necesarios para ser empleados en proyectos de pavimentación. Siendo una alternativa de solución mejorar las características mecánicas del material, estabilizándolo con productos adicionados, debido a que estudios sobre la estabilización de suelos han venido experimentando un importante crecimiento desde el año 2000(Morales, 2015, pág. 8)

La estabilización de suelos utilizando cenizas de carbón ha tenido óptimos resultados en nuestro país y en países como, Ecuador y Colombia ya que mejoraron sus propiedades mecánicas(Huancoillo, 2017, pág. 14).

En la periferia de la ciudad de Chachapoyas contamos con la apertura de muchas calles y dejadas a nivel de subrasante para la circulación vehicular y peatonal, sin tener en cuenta la deficiente capacidad de soporte con la que cuenta, debido a este problema se busca obtener un subproducto que ayude a mejorar dicha característica mecánica de los suelos y solucionar la problemática que se está viviendo.

Este trabajo, se centra en la estabilización de suelos con ceniza de carbón proveniente de la industria ladrillera, localizada en el distrito de Chachapoyas, con el fin de mejorar las características de soporte que ofrecen estos suelos a nivel de subrasante en el anexo 16 de Octubre; a la ceniza utilizada se denomina "CC" (Ceniza de Carbón) y los porcentajes (tratamientos) de CC empleadas en esta investigación fueron del 15\%, 20\% y $25 \%$.

El método empleado en el desarrollo de esta investigación implicó la realización de estudio de mecánica de suelo, mediante ensayos de laboratorio para obtener resultados físicos y mecánicos de las muestras de suelo extraídas de cada una de las calicatas. Para el procesamiento y análisis de los datos obtenidos de los ensayos de laboratorio, se tabularon, graficaron, se compararon mediante gráficos de barras y por último se utilizó el método estadístico diseño completamente al azar y la prueba de comparaciones múltiples (Diferencia Mínima Significativa) que ayudaron a determinar el tratamiento que presentó un mejor comportamiento en cada una de las muestras de suelo analizadas.

La capacidad de soporte se evaluó mediante ensayos de CBR, previo ensayo de compactación Proctor Estándar que ayudo a determinar el óptimo contenido de humedad y la máxima densidad seca, los materiales y equipos utilizados fueron proporcionados por el laboratorio de mecánica de suelos y concreto de la Dirección Regional de Transportes y Comunicaciones-Amazonas.

Se pretende que este trabajo sea una iniciativa para seguir evaluando a mayor profundidad el uso de las cenizas de carbón como material alternativo para la estabilización de suelos en vías no pavimentadas y contribuir con posteriores estudios.

\section{MATERIAL Y MÉTODOS}

\subsection{Localización de la investigación.}

Las muestras de suelo extraídas fueron de las cuadras ocho y nueve de calle Las Lomas, anexo 16 de octubre, distrito de Huancas, provincia Chachapoyas-Amazonas- Perú.

Los ensayos de esta investigación se realizaron en el laboratorio de mecánica de suelos y concreto de la Dirección Regional de Transportes y Comunicaciones - Amazonas (DRTC-A), ubicado en: Chachapoyas, Amazonas, Perú.

\subsection{Diseño de la investigación.}

Se aplicó el diseño experimental completamente al azar.

\subsection{Universo muestral.}

Para la presente investigación se extrajo muestras de suelo de las cuadras ocho y nueve de calle Las Lomas, así mismo se prepararon combinaciones de sueloceniza de carbón para ser sometido a ensayos mecánicos.

Para las muestras de suelo adicionadas cenizas de carbón del 15, 20 y 25\% de cenizas de carbón se realizó una repetición para los límites de consistencia y compactación Proctor estándar y cinco repeticiones para california bearing ratio (CBR).

\subsection{Técnicas e instrumentos}

- Técnicas de recolección: Observación.

- Instrumentos de recolección de información: Fichas técnicas de ensayos de laboratorio.

\subsection{Procedimiento}


Paso 1: Se extrajeron las muestras de suelo de (calle Las Lomas-16 de Octubre-Chachapoyas), para su posterior secado.

Paso 2: Recojo y tamizado por la malla $\mathrm{N}^{\circ} 4$ de las cenizas de carbón proveniente de la industria ladrillera.

Paso 3: Determinación de las características físicas de las muestras de suelo en estado natural, tales como:

- Humedad natural (MTC E 108/ASTM D2216)

- Análisis granulométrico por tamizado (MTC E 107/ASTM-D422, C-117/AASHTO T-27, T-88)

- Límites de consistencia (MTC E-110, 111/ ASTM D-4318/AASHTO T-90, T-89)

Límite líquido.

Límite plástico.

Índice de plasticidad.

Paso 4: Determinación de las características mecánica de las muestras de suelo en estado natural y adicionadas cenizas de carbón al 15\%, 20\% y 25\%, mediante ensayos de:

- Compactación proctor estandar (MTC E-115, E116/ASTM D-1557, D 698/AASHTO T-180)

- California bearing ratio (MTC E-132/ ASTM D1883/AASHTO T-193)

Paso 5: Se realizó el procesamiento de los datos que se recolectaron a partir de cada uno de los ensayos de laboratorio tanto para los suelos en estado natural, como para las muestras de suelo adicionadas cenizas de carbón.

Paso 6: Con el análisis de varianza y la prueba de diferencia mínima significativa se determinó el porcentaje de cenizas de carbón que tuvo un efecto significativo en el incremento de la capacidad de soporte para cada uno de los tipos de suelos estudiados.

\section{RESULTADOS}

\subsection{Resultados de ensayos de laboratorio}

\subsubsection{Humedad natural.}

Tabla 1. Contenido de humedad natural

\begin{tabular}{cc}
\hline CONTENIDO DE HUMEDAD (\%) \\
\hline $\mathrm{C}-1$ & 32.70 \\
\hline $\mathrm{C}-2$ & 28.31 \\
\hline
\end{tabular}

\subsubsection{Análisis granulométrico por tamizado}

La muestra de suelo de la calicata C-1 según SUCS pertenece al grupo $\mathrm{CH}$ siendo una arcilla inorgánica de alta plasticidad; según AASHTO pertenece al grupo A-7-6 (16) siendo un suelo arcilloso.
La muestra de suelo de la calicata C-2 según SUCS pertenece al grupo $\mathrm{OH}$ siendo una arcilla orgánica de alta o media

plasticidad; según AASHTO pertenece al grupo A-75 (13) siendo un suelo arcilloso.

\subsubsection{Límites de consistencia.}

Tabla 2. Resumen de los límites de consistencia de las calicatas 1 y 2 .

\begin{tabular}{crcc}
\hline \multirow{2}{*}{ MUESTRA } & \multicolumn{3}{c}{ LIMITES DE CONSISTENCIA } \\
\cline { 2 - 4 } & $\begin{array}{c}\text { Límite } \\
\text { líquido }\end{array}$ & $\begin{array}{c}\text { Límite } \\
\text { plástico }\end{array}$ & $\begin{array}{c}\text { Índice de } \\
\text { plasticidad }\end{array}$ \\
\hline C-1 & 51 & 27 & 24 \\
\hline C-1+15\%CC & 52 & 31 & 21 \\
\hline C-1+20\%CC & 53 & 34 & 19 \\
\hline C-1+25\%CC & 55 & 39 & 16 \\
\hline C-2 & 51 & 33 & 18 \\
\hline C-2+15\%CC & 52 & 35 & 17 \\
\hline C-2+20\%CC & 54 & 39 & 15 \\
\hline C-2+25\%CC & 55 & 42 & 13 \\
\hline
\end{tabular}

\subsubsection{Compactación proctor estándar}

Tabla 3. Óptimo contenido de humedad y máxima densidad seca calicata 1 y adición de cenizas de carbón $(15 \%, 20 \%$ y $25 \%)$.

\begin{tabular}{ccc}
\hline \multirow{2}{*}{ MUESTRA } & \multicolumn{2}{c}{ PROCTOR ESTANDAR } \\
\cline { 2 - 3 } & $\begin{array}{c}\text { óptimo } \\
\text { contenido de } \\
\text { humedad }(\%)\end{array}$ & $\begin{array}{c}\text { máxima } \\
\text { densidad seca } \\
\left(\mathrm{gr} / \mathrm{cm}^{3}\right)\end{array}$ \\
\hline $\mathrm{C}-1$ & 18.2 & 1.449 \\
\hline $\mathrm{C}-1+15 \% \mathrm{CC}$ & 19.1 & 1.457 \\
\hline $\mathrm{C}-1+20 \% \mathrm{CC}$ & 21.5 & 1.487 \\
\hline $\mathrm{C}-1+25 \% \mathrm{CC}$ & 24.7 & 1.494
\end{tabular}

Tabla 4. Óptimo contenido de humedad y máxima densidad seca, calicata 2 y adición de cenizas de carbón $(15 \%, 20 \%$ y $25 \%)$. 


\begin{tabular}{ccc}
\hline \multirow{2}{*}{ MUESTRA } & \multicolumn{2}{c}{ PROCTOR ESTANDAR } \\
\cline { 2 - 3 } & $\begin{array}{c}\text { óptimo } \\
\text { contenido } \\
\text { de } \\
\text { humedad } \\
(\%)\end{array}$ & $\begin{array}{c}\text { máxima } \\
\text { densidad seca } \\
\left(\mathrm{gr} / \mathrm{cm}^{3}\right)\end{array}$ \\
\hline $\mathbf{C - 2}$ & 21.3 & 1.473 \\
\hline $\mathbf{C - 2}+\mathbf{1 5 \% C C}$ & 26.5 & 1.525 \\
\hline $\mathbf{C - 2}+\mathbf{2 0} \% \mathbf{C C}$ & 26.7 & 1.551 \\
\hline $\mathbf{C}-2+\mathbf{2 5 \% C C}$ & 29.1 & 1.571 \\
\hline
\end{tabular}

\subsubsection{California bearing ratio (CBR)}

Tabla 5. Resumen de datos - CBR.

\begin{tabular}{|c|c|c|c|c|c|c|}
\hline \multirow{3}{*}{$\mathbf{C}$} & \multirow{3}{*}{$\begin{array}{c}\text { Adiciones } \\
\text { porcentua } \\
\text { les }\end{array}$} & \multicolumn{5}{|c|}{ CENIZAS DE CARBÓN } \\
\hline & & \multicolumn{5}{|c|}{ Observaciones } \\
\hline & & $1^{\text {ra }}$ & $2^{\mathrm{da}}$ & $3^{\mathrm{ra}}$ & $4^{\text {ta }}$ & $5^{\text {ta }}$ \\
\hline \multirow{4}{*}{1} & $0 \%\left(\mathrm{~T}_{1}\right)$ & 2.0 & 2.0 & 2.1 & 2.1 & 2.1 \\
\hline & $15 \%\left(\mathrm{~T}_{2}\right)$ & 2.1 & 2.2 & 2.1 & 2.4 & 2.5 \\
\hline & $20 \%\left(\mathrm{~T}_{3}\right)$ & 2.9 & 2.8 & 2.9 & 2.8 & 3.1 \\
\hline & $25 \%\left(\mathrm{~T}_{4}\right)$ & 3.5 & 3.5 & 3.4 & 3.3 & 3.6 \\
\hline \multirow{4}{*}{2} & $0 \%\left(\mathrm{~T}_{1}\right)$ & 2.1 & 2.2 & 2.2 & 2.2 & 2.2 \\
\hline & $15 \%\left(\mathrm{~T}_{2}\right)$ & 2.7 & 2.6 & 2.6 & 2.7 & 2.6 \\
\hline & $20 \%\left(\mathrm{~T}_{3}\right)$ & 2.9 & 3.0 & 2.9 & 3.0 & 3.0 \\
\hline & $25 \%\left(\mathrm{~T}_{4}\right)$ & 3.7 & 3.6 & 3.7 & 3.7 & 3.6 \\
\hline
\end{tabular}

**C: calicata

**T1: tratamiento 1; T2: tratamiento 2; T3: tratamiento 3; T4: tratamiento 4

En la Tabla 5 se tiene los valores del ensayo de CBR al $95 \%$ de la máxima densidad seca y a 0.1 " de penetración, de las muestras patrón y muestra con las tres adiciones porcentuales de 15,20 y $25 \%$ de cenizas de carbón de las dos calicatas estudiadas.

\subsection{Análisis de varianza.}

El factor calculado $(\mathrm{Fc}=126.79)$ mayor al factor de tabla $(\mathrm{F}(\mathrm{t}) 1 \%=5.29)$, por lo que los tratamientos son altamente significativos sobre el CBR para el tipo de suelo $\mathrm{CH}$ de la calicata 1 , con un coeficiente de variación de $4.74 \%$.

El factor calculado $(\mathrm{Fc}=703.52)$ mayor al $(\mathrm{F}(\mathrm{t}) 1 \%=$ $5.29)$, por lo que los tratamientos son altamente significativos sobre el CBR para el tipo de suelo $\mathrm{OH}$ de la calicata 2 , con un coeficiente de variación de $1.83 \%$.

\subsection{Prueba de Diferencia Mínima Significativa - Comparaciones Múltiples}

De las comparaciones múltiples entre combinaciones de tipo de suelo y porcentajes de cenizas de carbón, se determinó que $\mathrm{T} 4=25 \% \mathrm{CC}$ influye de manera significativa en el incremento del CBR.

Figura 1. CBR de las calicatas 1 y 2 adicionadas cenizas de carbón

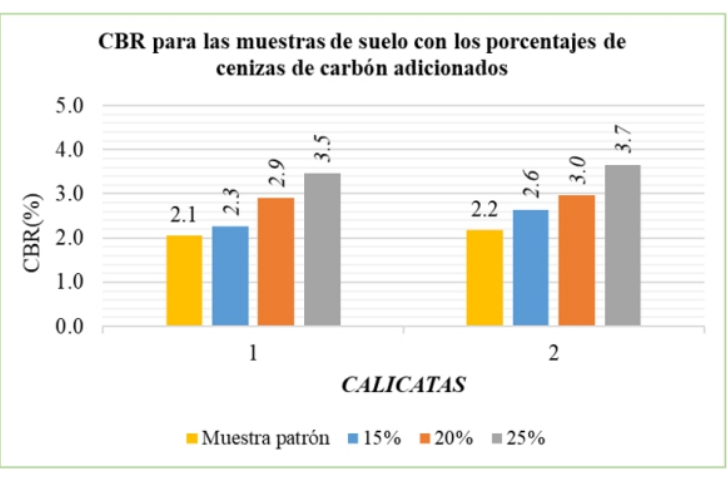

\section{DISCUSIÓN}

Del trabajo de investigación "Análisis comparativo de la resistencia al corte y estabilización de suelos arenosos finos y arcillosos combinadas con ceniza de carbón" (Cañar, 2017) el CBR que obtuvo para la muestra de suelo en estado natural para un tipo de suelo $\mathrm{CH}$ fue de $9.3 \%$ y cuando adicionó el 20 y $25 \%$ de cenizas de carbón los CBR fueron de 9.9\% y $10.9 \%$ respectivamente, del cual se observa que el incremento del CBR es de 0.60 al adicionarle el $20 \%$ y de 1.60 al adicionarle el $25 \%$ de cenizas de carbón; siendo un incremento de $6.45 \%$ y $17.20 \%$ respecto al CBR del suelo natural; al compararlo con los resultados de la tabla 14 de esta investigación el aumento es de 0.80 al adicionarle el $20 \%$ y del 1.4 al agregarle el $25 \%$ de cenizas de carbón lo cual representa el $38.09 \%$ y $66.67 \%$ de incremento respecto al valor inicial de CBR de las muestras sin la adición de cenizas de carbón; por lo que Cañar concluye que la adición de las cenizas de carbón influye favorablemente en la estabilización de suelos expansivos y por lo tanto mejora su CBR, hecho que se confirma con esta investigación ya que se logró mejorar las propiedades mecánicas de los suelos en estudio.

En la tesis "Estabilización de suelos arcillosos con cenizas de carbón para su uso como subrasante mejorada y/o sub base de pavimentos" (Pérez, 2012), indica que el CBR tuvo un incremento de 9.2 al agregarle $20 \%$ de cenizas volantes de carbón siendo un incremento de $119.48 \%$ respecto al CBR del suelo natural para un tipo de suelo $\mathrm{CH}$; sin embargo en esta investigación el incremento del CBR al adicionarle el $20 \%$ de cenizas de carbón es de 0.80 el cual 
representa el $38.09 \%$ de incremento respecto al valor inicial del CBR de la muestra sin adicionarle cenizas de carbón; de los resultados concluimos que los incrementos de CBR obtenido por Pérez es mucho mayor a los que se lograron en esta tesis, esto debido al tipo de ceniza utilizada como material estabilizante.

De la tesis "Estabilización de suelos arcillosos con cenizas de carbón para su uso como subrasante mejorada" (Peréz, 2014), menciona que el CBR aumentó considerablemente de $8.3 \%$ a $17.3 \%$ adicionándole la ceniza volante en la proporción de $20 \%$, siendo este un incremento de $108.43 \%$ con respecto al suelo en estado natural para un suelo arcilloso, concluyendo que la ceniza volante mejoró las propiedades de resistencia y de cohesión en las arcillas. Los resultados que se obtuvieron en esta investigación con la adición del $20 \%$ de cenizas de carbón fueron muy inferiores a los resultados de la tesis mencionada, ya que el CBR aumento de $2.1 \%$ a $2.9 \%$ con la adición del $20 \%$ de cenizas de carbón, siendo un incremento porcentual de $38.09 \%$; esta gran diferencia se debe al tipo de ceniza que se utilizó para cada una de las investigaciones.

De la misma manera se encuentra semejanza con lo que sostiene (Cubas \& Falen, 2016) donde concluye que las cenizas de carbón e $\mathrm{NaOH}$ puede ser usado para la estabilización de suelos arenosos pobremente graduados con arcillas para mejorar la subrasante, de la misma manera(Gonzáles, 2014) concluye que la utilización de cal y ceniza volante para la estabilización de suelo cohesivo es eficaz, por generar un mejoramiento de hasta cuatro veces el valor inicial de CBR a 95 por ciento.

\section{CONCLUSIONES}

Las cenizas de carbón mejoran las propiedades mecánicas (CBR) de los suelos tipo $\mathrm{CH}$ y $\mathrm{OH}$, aunque no alcanzan los estándares para ser usadas como material apto como subrasante debido a que se obtuvieron valores de CBR de $3.5 \%$ y $3.7 \%$ respectivamente, sin superar el valor mínimo de $6 \%$ según lo indica el manual de carreteras "Suelos, Geología, Geotecnia y Pavimentos" Sección: Suelos y Pavimentos.

La capacidad de soporte (CBR) obtenidos sin la adición de cenizas de carbón de las muestras de las calicatas 1 y 2 a nivel de subrasante fueron de $2.1 \%$ y $2.2 \%$ respectivamente.

La capacidad de soporte (CBR) obtenidos fueron de: $2.3 \%, 2.9 \%$ y $3.5 \%$ con la adición de cenizas de carbón del 15\%, 20\% y 25\% respectivamente para el tipo de suelo $\mathrm{CH}$ de la calicata 1; para la calicatas 2, tipo de suelo $\mathrm{OH}$, la capacidad de soporte (CBR) obtenidos fueron de: $2.6 \%, 3.0 \%$ y $3.7 \%$ con la adición de cenizas de carbón del 15\%, 20\% y 25\% respectivamente.

La capacidad de soporte (CBR) obtenidos fueron de: $2.3 \%, 2.9 \%$ y $3.5 \%$ con la adición de cenizas de carbón del $15 \%, 20 \%$ y $25 \%$ respectivamente para el tipo de suelo $\mathrm{CH}$ de la calicata 1; para la calicatas 2, tipo de suelo $\mathrm{OH}$, la capacidad de soporte (CBR) obtenidos fueron de: $2.6 \%, 3.0 \%$ y $3.7 \%$ con la adición de cenizas de carbón del 15\%, 20\% y 25\% respectivamente.

Estadísticamente se determinó que la adición del $25 \%$ de cenizas de carbón proporciona un mejor comportamiento a la subrasante de los suelos tanto para la calicata 1 y la calicata 2 .

\section{REFERENCIAS BIBLIOGRÁFICAS}

Badillo J. (2002). Mecánica de suelos. Mexico.

Cañar, E. (2017). Análisis comparativo de la resistencia al corte y estabilización de suelos arenosos finos y arcillosos combinados con cenizas de carbón. Trabajo experimental previo a la optención del título de ingeniero civil. Universidad Técnica de Ambato, Ambato, Ecuador.

Cubas, K., \& Falen, J. (2016). Evaluación de las cenizas de carbón para la estabilización de suelos mediante activación alcalina y aplicación en carreteras no pavimentadas. Tesis para optar el título de ingeniero civil. Universidad Señor de Sipan, Pimentel, Perú.

Gonzáles, A. (2014). Estabilización mecánica de suelos cohesivosa través de la utilización de cal-cenizas volantes. Trabajo de grado para optar el grado de ingeniero civil. Universidad de San Carlos de Guatemala, Guatemala, Guatemala.

Huancoillo, Y. (2017). Mejoramiento de suelo arcilloso con ceniza volante y cal para su uso como pavimento a nivel de afirmado en la carretera desvio Huancané -Chupa Puno. Tesis para optar el título profesional de ingeniero civil. Universidad Nacional del Altiplano, Puno, Perú.

Badillo J. (2002). Mecánica de suelos. Mexico.

Cañar, E. (2017). Análisis comparativo de la resistencia al corte y estabilización de suelos arenosos finos y arcillosos combinados con cenizas de carbón. Trabajo experimental previo a la optención del título de ingeniero civil. Universidad Técnica de Ambato, Ambato, Ecuador. 
Cubas, K., \& Falen, J. (2016). Evaluación de las cenizas de carbón para la estabilización de suelos mediante activación alcalina y aplicación en carreteras no pavimentadas. Tesis para optar el título de ingeniero civil. Universidad Señor de Sipan, Pimentel, Perú.

Gonzáles, A. (2014). Estabilización mecánica de suelos cohesivosa través de la utilización de cal-cenizas volantes. Trabajo de grado para optar el grado de ingeniero civil. Universidad de San Carlos de Guatemala, Guatemala, Guatemala.

Huancoillo, Y. (2017). Mejoramiento de suelo arcilloso con ceniza volante y cal para su uso como pavimento a nivel de afirmado en la carretera desvío Huancané -Chupa Puno. Tesis para optar el título profesional de ingeniero civil. Universidad Nacional del Altiplano, Puno, Perú.

Ministerio de Transportes y comunicaciones. (2014). Manual de carreteras, Suelos, geologia, geotécnia y pavimentos. Lima, Perú.

Ministerio de Transportes y Comunicaciones. (mayo de 2016). Manual de ensayo de materiales. Lima, Perú.

Morales, D. (2015). Valoración de las cenizas de carbón para la estabilización de Suelos mediante activación alcalina y su uso en vias no pavimentadas. Trabajo de grado para optar al titulo de ingeniero civil. Universidad de Medellín, Medellín, Colombia.

Peréz, C. (2014). Estabilizacion de suelos arcillosos con cenizas de carbón para su uso como subrasante mejorada. Tesis para optar el titulo profesional de ingeniero civil. Universidad Nacional de Ingeniería, Lima, Perú.

Peréz, R. (2012). Estabilización de suelos arcillosos con cenizas de carbón para su uso como subrasante mejorada y/o sub base de pavimentos. Tesis para optar el grado de maestro en ciencias con mencion en ingeniería geotécnica. Universidad Nacional de Ingeniería, Lima, Perú 